\title{
Compressive and Flexural Strengths of High-strength Glass Ionomer Cements: A Systematic Review
}

\author{
Ridyumna Garain ${ }^{1}$, Mikayeel Abidi², Zain Mehkri
}

\begin{abstract}
Objective:To perform a systematic review of test methodologies of high-strength restorative glass ionomer cement (GIC) materials for compressive (CS) and flexural strengths (FS) to compare the results between different GICs.

Materials and methods: Screening of titles and abstracts, data extraction, and quality assessment was conducted in search for in vitro studies, which reported on CS and/or FS properties of high-strength GIC. PubMed/Medline (US National Library of Medicine-National Institutes of Health), EBSCO, and ProQuest databases were searched for the relevant literature.

Results: A total of 123 studies were found. These were then assessed based on preestablished inclusion and exclusion criteria. Of the selected studies, two studies of fair quality tested CS, while none tested FS. The CS of experimental groups in both studies was less than their respective control groups.

Discussion: It was observed that many studies reported following the International Standards Organization (ISO) recommendations for testing but with modifications. Additionally, in absence of guidelines for testing other parameters that may be potentially advantageous, authors have used differing experimental techniques. These disparities make it difficult to draw comparisons between different studies.

Conclusion: Only two studies of fair quality showed lower CS of experimental groups compared to their respective control groups. Lack of adherence to guidelines and lack of guidelines for potentially better test methodologies make it difficult to scrutinize and compare the validity of the research being conducted.
\end{abstract}

Keywords: Compressive strength, Flexural strength, Glass ionomer cement, High strength GIC, Materials testing, Review, Standards. International Journal of Experimental Dental Science (2020): 10.5005/jp-journals-10029-1207

\section{INTRODUCTION}

Glass ionomer cements (GICs) were invented in 1969 and their use was reported by Wilson and Kent in the early 1970s. They are composed of an aluminosilicate glass and a polyalkenoic acid.

Glass ionomer cements possess unique properties such as biocompatibility, elasticity similar to dentin, anticariogenic action due to fluoride release, and direct bonding to the tooth structure. Conversely, GICs have also presented clinical limitations such as low wear resistance, low fracture toughness, low mechanical properties, prolonged setting rate, and high early moisture sensitivity. ${ }^{1,2}$ These limitations have restricted the use of conventional GICs in high stress-bearing areas (e.g., posterior teeth).

High-strength/highly viscous GICs have since been developed to address the relatively poor physical and mechanical properties of conventional GICs and to be used as restorative materials in higher stress-bearing posterior teeth. However, these materials may still experience restoration failures, e.g., in multiple-surface atraumatic restorative technique (ART) restorations, restorations in high stressbearing sites, ${ }^{3,4}$ indicating the need for further improvement in their physical and mechanical properties.

Efforts have been made toward improving high-strength GICs' physical and mechanical properties without affecting their biological properties, by the addition of a variety of filler materials including montmorillonite clay, ${ }_{1}^{5}$ zirconia, ${ }^{6}$ glass fibers, ${ }^{7}$ hydroxyapatite, bioactive glass particles as prereacted glass ionomer particles, and changing the type of alkenoic acid..$^{8,9}$

With the wide variety of dental products constantly being launched, selecting an ideal restorative material has become a difficult task for the clinician. Prior to clinical trials, laboratory tests \begin{tabular}{l}
\hline \hline Department of Conservative Dentistry and Endodontics, Dayananda \\
Sagar College of Dental Sciences, Bengaluru, Karnataka, India \\
2,3 Dayananda Sagar College of Dental Sciences, Bengaluru, Karnataka, \\
India \\
Corresponding Author: Ridyumna Garain, Department of \\
Conservative Dentistry and Endodontics, Dayananda Sagar College of \\
Dental Sciences, Bengaluru, Karnataka, India, Phone: +919113917831, \\
e-mail: garain.ridyumna@gmail.com \\
How to cite this article: Garain R, Abidi M, Mehkri Z. Compressive \\
and Flexural Strengths of High-strength Glass lonomer Cements: A \\
Systematic Review. Int J Experiment Dent Sci 2020;9(1):25-29. \\
Source of support: Nil \\
Conflict of interest: None \\
\hline \hline
\end{tabular}

are a fundamental necessity in guiding researchers and clinicians to analyze and understand the factors affecting the variability in test results of a material, thus facilitating evidence-based practice.

However, many of the studies that have evaluated the properties of high-strength GICs have shown variability in protocols, in terms of temperature of specimen storage, duration of storage, dimensions of the specimens, and the load applied during tests. This lack of uniform methods and reporting hampers meaningful comparisons of these studies. 9,10

Thus, in view of the new materials being developed, it is necessary to verify the standardization of laboratory tests performed with these materials so that future manufacturers, clinicians, and researchers can rely on in vitro studies to deduce inferences. 
Therefore, the objectives of this review are the following:

- To systematically analyze and compare the test methodologies employed for testing the compressive strength (CS) and flexural strength (FS) for new/experimental high-strength GICs for compliance with the recommendations of testing established by the ISO.

- To compare the above-mentioned properties of different GICs that have been tested according to the International Standards Organization (ISO) recommendations.

- To assess the quality of evidence of these studies.

\section{Materials and Methods Eligibility Criteria Inclusion Criteria} In vitro studies published from January 1, 1990 to December 31, 2019, which reported on mechanical (CS and/or FS) properties of highstrength posterior GICs for dental use, were considered eligible.

\section{Exclusion Criteria}

Studies were excluded, if:

- The use of conventional restorative GIC was reported;

- The use of resin-modified GIC was reported;

- The use of GIC other than high-strength GIC meant for posterior restorations or ART was reported;

- The focus of the study was for the use of GIC in fields other than dentistry;

- The use of light or heat-curing was reported; and

- There was incorrect or missing statistical analysis.

\section{Databases and Search Strategy}

The literature search was conducted on the PubMed/Medline (US National Library of Medicine-National Institutes of Health), EBSCO, and ProQuest databases.

The following search criteria were employed as the keywords to search for relevant articles in the mentioned databases:

(Posterior OR (High strength) OR ((ART) OR (Atraumatic Restorative))) AND ((Glass AND ionomer OR (polyalkenoate) AND (cement))) AND ((Mechanical properties) OR (Compressive OR compression AND strength) OR (Flexural OR Flexion OR Flexure AND Strength)) NOT (Resin).

\section{Selection of Studies}

Abstracts and titles of the studies identified by the search strategy were screened by the investigators to select the studies that would be fully read. The selected studies were then analyzed to check their eligibility according to the inclusion and exclusion criteria. In case of disagreement between the investigators, a consensus was reached through discussion with external consultation. Following the eligibility check, the eligible studies were checked for compliance with ISO recommendations for compressive and three-point flexural strength (TFS) testing as per ISO 9917-1:2007 ${ }^{11}$ and ISO 9917-2:2010, ${ }^{12}$ respectively.

\section{Risk of Bias and Quality of Evidence}

Data extraction and quality assessment of the studies that adhered to ISO recommendations were carried out. The CRIS guidelines ${ }^{13}$ for in vitro studies were followed for the quality assessment by analyzing the following variables: (1) sample preparation and handling; (2) allocation sequence and randomization process; (3) whether the evaluators were blinded, and (4) statistical analysis. Studies providing information of all variables were considered to be of good quality; if two to three variables were present, they were considered of fair quality; and last, studies were classified as being of poor quality when none or just one variable was reported.

Flowchart 1 depicts a flowchart of the methodology followed.

\section{Results}

Initially, 123 studies were found, from which 115 were selected after removing 8 duplicates. These 115 studies were then screened by reading the titles and abstracts, and 49 were selected. The full-text of these 49 studies was accessed, and 23 studies were excluded based on the exclusion criteria; 26 studies were included. These 26 studies were checked for compliance with ISO recommendations, and 2 studies were found to comply with the ISO recommendations for testing. Flowchart 1 depicts this process.

The number of studies not complying with the ISO recommendations is shown in Table 1. It was observed that only two studies evaluating CS complied with ISO recommendations, while none studying FS were compliant with the ISO recommendations. The characteristics of these studies are displayed in Table 2.

With respect to the quality assessment, both studies were considered of fair quality. The studies did not provide information pertaining to how the samples were allocated to the different groups or blinding of evaluators to the type of material or procedure.

\section{Discussion}

A previous systematic review on mechanical and optical properties of conventional GICs found that only one study complied with the ISO recommendations CS testing. ${ }^{16}$ Similar results of this review indicate that there is a need for researchers to reconsider the test methodologies that they follow for uniform reporting and better interpretation of results.

From the results of this review, depending on the property being tested, only two or no studies followed the recommendations laid down by the ISO. Many of the studies reported having followed the specifications but with modifications in dimensions, storage media/conditions, storage time, etc., of the specimens. Studies have demonstrated that CS can be influenced by specimen dimensions as well as storage media and testing conditions. ${ }^{17-19}$ While the ISO provides guidance for the evaluation of dental materials with the intention of ensuring reproducibility of test results for selected testing methodologies between different test centers under standard conditions, such modifications make it difficult to draw comparisons.

Only two studies in this review adhered to the ISO recommendations for CS testing of GICs.

Both the studies were judged to be of fair quality according to the CRIS guidelines for in vitro studies as they did not provide information about randomization/sample allocation and investigator blinding. Randomization in in vitro experiments reduces the chances of bias and favors the difference in outcome between groups to be by chance, just as in randomized clinical trials. Reporting sample size calculation, meaningful difference, and randomization would make results of in vitro studies more robust and significant. ${ }^{13}$

The study by Yesilyurt et al. ${ }^{14}$ evaluated the antibacterial activity and physical properties (CS and bond strength to dentin) of GICs 
Flowchart 1: PRISMA flowchart showing the methodology of the review

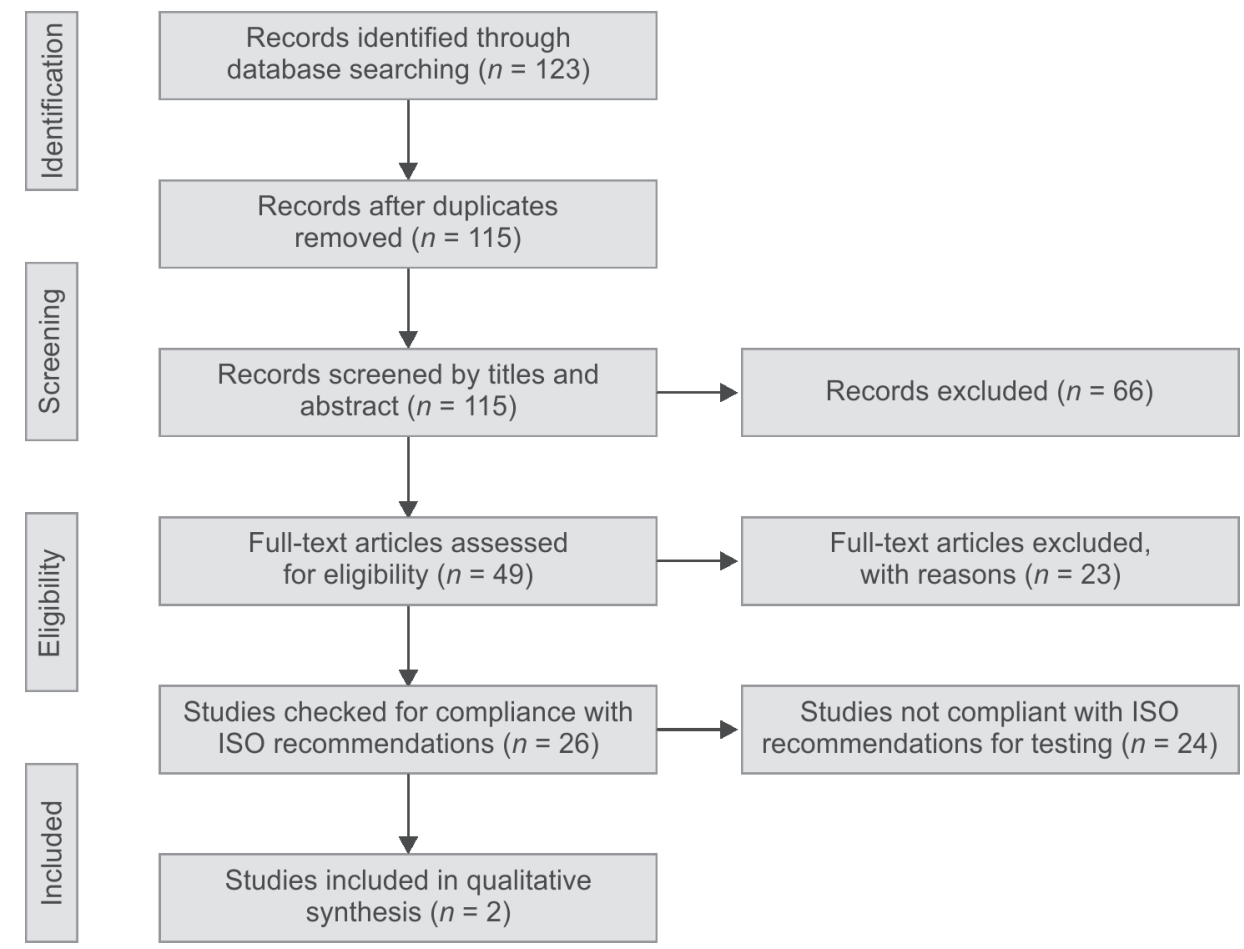

Table 1: Studies not complying with ISO recommendations by property

\begin{tabular}{lrr}
\hline Property & CS & FS \\
\hline Storage media and conditions & 14 & 14 \\
Storage time & 7 & 8 \\
Specimen dimensions & 10 & 3 \\
Cross-head speed & 2 & 1 \\
\hline
\end{tabular}

containing an antibiotic mixture of ciprofloxacin, metronidazole, and minocycline in concentrations of $1.5,3.0$, and $4.5 \% \mathrm{w} / \mathrm{w}$ for use with ART. The results of this study showed that the experimental GICs were effective in inhibiting bacteria associated with caries. They also displayed reduced bond strength to dentin. At the end of 24 hours, all the experimental groups as well as the control groups showed CS lower than that specified as the minimum required CS by the ISO, i.e., $100 \mathrm{MPa}$. The ISO specifies that at least four out of five test results should be above the minimum specified strength. If three or more of five results are below the specified minimum strength, the material fails the test, in which case, another batch of five specimens must be tested. Subsequently, at least 8 out of a total of 10 results must be above the minimum specified strength. It has been suggested that at least 20 similar specimens are required to determine statistically significant differences when assessing the CS of brittle dental materials. ${ }^{20,21}$ However, this study considered a sample size of 5 per group for CS testing. According to the authors, a possible explanation for these results is that increasing concentration of antibiotic powders may contribute to incomplete reactions between the glass particles and liquid, thereby increasing the number of unreacted particles in the structure. The powdered antibiotic particles used in this study were hygroscopic. Absorption of water by these additives could be a contributory factor to the decrease in the CSs of the GIC. The low CS of these GICs may make them unsuitable for use as materials in posterior teeth for ART.
The second study, by El-Wassefy et al., ${ }^{15}$ studied the effect of incorporating silver nanoparticles in concentrations of $0,1,3$, and $5 \mathrm{wt} \%$ to GIC on the Staphylococcus aureus biofilm, in terms of bacterial growth and effect on hardness and CS. The CS reported by the study was more than the minimum specified strength by the ISO. However, there was a decrease in the CS of the experimental GICs, which was considered to be statistically insignificant. A possible explanation for this, as given by the authors, is that the nanosize of the silver nanoparticles and physical addition instead of bonding the particles to the matrix did not alter the mechanical properties significantly. The nanosize of the particles allows the dispersion in-between and around polymer chains. It is also assumed that silver does not interfere with the matrix setting reactions. The authors concluded that the incorporation of silver nanoparticles in GIC powder could limit the formation of biofilm with a nonsignificant effect on the mechanical properties. However, the influence of silver nanoparticles on color was noted, which would restrict its use to areas where esthetics is not of concern.

Despite CS being considered as one of the parameters for evaluating strength by the ISO, its validity has been questioned. ${ }^{22,23}$ A major criticism of the CS testing methodology is that CS testing provides the stress at failure and neglects the mechanism of failure. Uniaxially compressed cylinders fail due to "some unresolved combination of tension and shear" stresses. The CS data do not help to determine "service performance."22

Alternatives to CS for GICs are the TFS and the biaxial flexure strength (BFS) test. ${ }^{21}$ The ISO 9917-2 provides specifications for the TFS testing of GICs, and none of the studies in this review complied with ISO specifications for TFS testing.

However, researchers have reported BFS testing to be more advantageous over uniaxial tests such as TFS because the specimens are considered to be easier to prepare and closely match the volume of a clinical restoration. The BFS testing also creates a 
Table 2: Characteristics of studies compliant with ISO recommendations

\begin{tabular}{|c|c|c|c|c|c|}
\hline Reference & Materials & Modifiers & Properties tested & Sample size per group & Outcomes (CS) \\
\hline Yesilyurt et al. ${ }^{14}$ & Fuji IX & $\begin{array}{l}\text { Ciprofloxacin, } \\
\text { metronidazole, } \\
\text { minocycline, }\end{array}$ & $\begin{array}{l}\text { CS, Shear bond strength, } \\
\text { antimicrobial activity screening, } \\
\text { antibacterial release }\end{array}$ & 5 & $\begin{array}{l}\text { Experimental groups showed } \\
\text { lower CS when compared with } \\
\text { the control group }\end{array}$ \\
\hline El-Wassefy et al. ${ }^{15}$ & Fuji IX & Ag nanopowder & $\begin{array}{l}\text { CS, microhardness, biofilm } \\
\text { formation, minimum inhibitory } \\
\text { concentration }\end{array}$ & 20 & $\begin{array}{l}\text { No significant decrease in } \\
\text { CS between control and } \\
\text { experimental groups, and } \\
\text { higher than ISO specification of } \\
\text { minimum CS of } 100 \mathrm{MPa}\end{array}$ \\
\hline
\end{tabular}

stress field away from the supports and away from the specimen periphery, reducing the sensitivity to specimen edge defects. ${ }^{10}$

In the absence of strict guidelines for testing other strength parameters that may be potentially advantageous over the existing ones in use, different authors have used different experimental techniques, ${ }^{10}$ which again makes it difficult to draw comparisons.

To summarize, despite the large number of studies that have been conducted on high-strength posterior GICs, lack of adherence to guidelines makes it difficult to compare results of different studies. Lack of guidelines for potentially better test methodologies and guidelines for test methods that may not be as apt make it difficult to scrutinize and compare the validity of the research being conducted.

\section{CONCLUSION}

The evidence from this review on the CS and FS of high-strength posterior GIC indicates that:

- Only two published studies that tested the CS of high-strength posterior GIC followed the ISO standards. Both studies were considered of fair quality.

- Both studies showed a decrease in the CS of the experimental groups compared to the control groups.

- None of the studies that tested FS adhered to the ISO recommendations.

There is a need for researchers to adhere to the guidelines laid down by the ISO to ensure validity and comparability of the data.

There is a need for ISO to reconsider the parameters that are currently in use in favor of potentially more advantageous parameters.

Adhering to a checklist or guidelines similar to CRIS guidelines for in vitro studies would make reporting of in vitro studies more significant and reliable.

\section{References}

1. Pereira LC, Nunes MCP, Dibb RGP, et al. Mechanical properties and bond strength of glass-ionomer cements. J Adhes Dent [Internet] 2002;4(1):73-80. Available from: http://www.ncbi.nlm.nih.gov/ pubmed/12071632.

2. Meşe A, Burrow MF, Tyas MJ. Sorption and solubility of luting cements in different solutions. Dent Mater J 2008;27(5):702-709. DOI: 10.4012/ dmj.27.702.

3. de Amorim RG, Leal SC, Frencken JE. Survival of atraumatic restorative treatment (ART) sealants and restorations: a meta-analysis. Clin Oral Investig [Internet] 2012;16(2):429-441.DOI: 10.1007/s00784-011-0513-3. Available from: http://www.ncbi.nlm.nih.gov/pubmed/21274581.

4. Zanata RL, Fagundes TC, de Almendra Freitas MCC, et al. Ten-year survival of ART restorations in permanent posterior teeth. Clin Oral Investig [Internet] 2011;15(2):265-271. DOI: 10.1007/s00784-009- 0378-x. Available from: http://link.springer.com/10.1007/s00784-0090378-x.

5. Dowling $A H$, Stamboulis A, Fleming GJP. The influence of montmorillonite clay reinforcement on the performance of a glass ionomer restorative. J Dent [Internet] 2006;34(10):802-810. DOI: 10.1016/j.jdent.2006.03.005. Available from: https://linkinghub. elsevier.com/retrieve/pii/S0300571206000686.

6. GuYW, Yap AUJ, Cheang P, etal. Development of zirconia-glassionomer cement composites. J Non Cryst Solids [Internet] 2005;351(6-7): 508-514. DOI: 10.1016/j.jnoncrysol.2005.01.045. Available from: http://linkinghub.elsevier.com/retrieve/pii/S0022309305000682.

7. Hammouda IM. Reinforcement of conventional glass-ionomer restorative material with short glass fibers. J Mech Behav Biomed Mater [Internet] 2009;2(1):73-81. DOI: 10.1016/j.jmbbm.2008.04.002. Available from: http://www.ncbi.nlm.nih.gov/pubmed/ 19627810.

8. Smith DC. 1st European Union Conference On Glass-lonomers, UK, 14-16 May 1997: development of glass-ionomer cement systems. Biomaterials [Internet] 1998;19(6):467-478. DOI: 10.1016/S01429612(97)00126-9. Available from: http://www.ncbi.nlm.nih.gov/ pubmed/9645552.

9. Baig MS, Fleming GJP. Conventional glass-ionomer materials: a review of the developments in glass powder, polyacid liquid and the strategies of reinforcement. J Dent [Internet]. 2015;43(8):897-912. DOI: 10.1016/j.jdent.2015.04.004.

10. Dowling AH, Fleming GJP, McGinley EL, et al. Improving the standard of the standard for glass ionomers: an alternative to the compressive fracture strength test for consideration? J Dent [Internet]. Elsevier Ltd; 2012;40(3):189-201. DOI: 10.1016/j.jdent.2011.12.002.

11. International Organization of Standardization. ISO 9917-1:2007 - dental-water-based cements. Part 1: Powder/liquid acid-base cements. Geneva: ISO; 2016.

12. International Organization for Standardization. ISO 9917-2:2010Dentistry - water based cements. Part 2: Resin-modified cements. Geneva: ISO; 2017.

13. Krithikadatta J, Gopikrishna V, Datta M. CRIS guidelines (checklist for reporting in-vitro studies): a concept note on the need for standardized guidelines for improving quality and transparency in reporting in-vitro studies in experimental dental research. J Conserv Dent 2014;17(4):301-304. DOI: 10.4103/0972-0707. 136338.

14. Yesilyurt C, Er K, Tasdemir T, et al. Antibacterial activity and physical properties of glass-ionomer cements containing antibiotics. Oper Dent 2009;34(1):18-23. DOI: 10.2341/08-30.

15. El-Wassefy NA, El-Mahdy RH, El-Kholany NR. The impact of silver nanoparticles integration on biofilm formation and mechanical properties of glass ionomer cement. J Esthet Restor Dent 2018;30(2):146-152. DOI: 10.1111/jerd.12353.

16. Menezes-Silva R, Cabral RN, Pascotto RC, et al. Mechanical and optical properties of conventional restorative glassionomer cements-a systematic review. J Appl Oral Sci 2019;27:1-9. DOI: 10.1590/16787757-2018-0357.

17. Mallmann A, Ataíde JCO, Amoedo R, et al. Compressive strength of glass ionomer cements using different specimen dimensions. Braz Oral Res [Internet] 2007;21(3):204-208. DOI: 10.1590/S1806- 
83242007000300003. Available from: http://www.ncbi.nlm.nih.gov/ pubmed/17710284.

18. Nicholson JW, Abiden F. Changes in compressive strength on ageing in glass polyalkenoate (glass-ionomer) cements prepared from acrylic/maleic acid copolymers. Biomaterials 1997;18(1):59-62. DOI: 10.1016/S0142-9612(96)00109-3.

19. Williams JA, Billington RW. Changes in compressive strength of glass ionomer restorative materials with respect to time periods of $24 \mathrm{~h}$ to 4 months. J Oral Rehabil 1991;18(2):163-168. DOI: 10.1111/j.13652842.1991.tb00044.x.

20. McCabe JF, CarrickTE. A statistical approach to the mechanical testing of dental materials. Dent Mater 1986;2(4):139-142. DOI: 10.1016/ S0109-5641(86)80021-5.
21. Fleming GJP, Dowling $A H$, Addison $O$. The crushing truth about glass ionomer restoratives: Exposing the standard of the standard. J Dent [Internet] 2012;40(3):181-188. Available from: http://dx.doi. org/10.1016/j.jdent.2011.12.004.

22. Darvell BW. Uniaxial compression tests and the validity of indirect tensile strength. J Mater Sci [Internet] 1990;25(2A):757780. Available from: http://link.springer.com/10.1007/ BF03372161.

23. McCabe JF, Watts $D C$, Wilson $\mathrm{HJ}$, et al. An investigation of testhouse variability in the mechanical testing of dental materials and the statistical treatment of results. J Dent 1990;18(2):90-97. DOI: 10.1016/0300-5712(90)90091-R. 